\title{
High-Fructose Diet-Induced Hypertriglyceridemia Is Associated With Enhanced Hepatic Expression of ACAT2 in Rats
}

\author{
Y. ICHIGO ${ }^{1}$, A. TAKESHITA ${ }^{2}$, M. HIBINO ${ }^{2}$, T. NAKAGAWA ${ }^{1,2}$, T. HAYAKAWA $^{1,2}$, \\ D. PATEL ${ }^{3}$, C. J. FIELD ${ }^{3}$, M. SHIMADA ${ }^{1,2,3}$
}

${ }^{1}$ Division of Life Science for Food, Department of Life Science and Chemistry, Graduate School of Natural Science and Technology, Gifu University, Yanagido, Gifu, Japan, ${ }^{2}$ Department of Applied Life Science, Faculty of Applied Biological Sciences, Gifu University, Yanagido, Gifu, Japan, ${ }^{3}$ Department of Agricultural, Food and Nutritional Science, Li Ka Shing Center for Health Research Innovation, University of Alberta, Edmonton, Canada

Received June 3, 2019

Accepted August 8, 2019

Epub Ahead of Print October 25, 2019

\begin{abstract}
Summary
High levels of fructose induce hypertriglyceridemia, characterized by excessive levels of triglyceride-rich lipoproteins such as very low-density lipoprotein (VLDL); however, the underlying mechanisms are poorly understood. The aim of this short communication was to examine hepatic changes in the expression of genes related to cholesterol metabolism in rats with hypertriglyceridemia induced by high-fructose or high-glucose diets. Rats were fed a $65 \%(w / w)$ glucose diet or a $65 \%(w / w)$ fructose diet for 12 days. Serum levels of triglycerides, total cholesterol, and VLDL+LDL-cholesterol, hepatic levels of triglycerides and cholesterol, and ACAT2 expression at the gene and protein levels were significantly higher in the fructose diet group compared to the glucose diet group. The hepatic levels of $A b c g 5 / 8$ were lower in the fructose group than in the glucose group. Serum high-density lipoprotein (HDL)-cholesterol and hepatic expression levels of Hmgcr, Ldlr, Acat1, Mttp, Apob, and Cyp7a1 did not differ significantly between groups. These findings suggest that high-fructose diet-induced hypertriglyceridemia is associated with increased hepatic ACAT2 expression.
\end{abstract}

\section{Key words}

ACAT2 • Fructose $\bullet$ Hypertriglyceridemia $\bullet$ Liver $\bullet$ Rats

\section{Corresponding author}

M. Shimada, Department of Applied Life Science, Faculty of Applied Biological Sciences, Gifu University, 1-1 Yanagido, Gifushi, Gifu 501-1193, Japan. E-mail: mshimada@gifu-u.ac.jp
Fructose is mainly consumed as dietary sugars (e.g., sucrose or high-fructose corn syrup) and its excessive consumption is closely related to metabolic diseases such as fatty liver and obesity (Herman and Samuel 2016). Several animal studies including ours have demonstrated that fructose, compared to that with glucose, potently induces the de novo synthesis of fatty acids and triglyceride accumulation in the liver (Koo et al. 2008, Janevski et al. 2012, Shimada et al. 2017, 2019) most likely due to bypassing the main rate-limiting step in glycolysis. Excessive triglycerides are assembled with cholesterol and apolipoproteins in the liver and are secreted as very low-density lipoprotein (VLDL) particles into the blood. Thereby, excessive intake of fructose, compared to that of glucose, leads to hypertriglyceridemia. However, the mechanisms through which fructose induces hypertriglyceridemia including VLDL secretion are poorly understood. Little evidence is available regarding the relationship between a highfructose or a high glucose diet and hepatic cholesterol metabolism. Koo et al. (2008) showed that high levels of fructose, as opposed to glucose, do not enhance the expression of 3-hydroxy-3-methylglutaryl-CoA reductase (Hmgcr) and microsomal triglyceride transfer protein (Mttp) genes in rat livers despite hypertriglyceridemia induced by high fructose. These genes encode the rate limiting enzyme for cholesterol synthesis (HMGCR) and 
a protein involved in the assembly and secretion of apolipoprotein B (APOB)-containing lipoproteins that stabilize VLDL (MTTP). Therefore, we hypothesized that high fructose-induced hypertriglyceridemia might affect other genes related to cholesterol metabolism in the liver. In this study, we examined changes in the hepatic expression of genes related to cholesterol synthesis/uptake/secretion/catabolism/excretion in rats with hypertriglyceridemia induced by a high-fructose diet, as compared to that with a high-glucose diet.

Five-week-old male Wistar rats (SLC, Shizuoka, Japan) were assigned to a glucose diet group or a fructose diet group ( $\mathrm{n}=7$ per group). The composition of diets was as follows: $20.0 \% \quad(\mathrm{w} / \mathrm{w})$ casein, $65.0 \%$ carbohydrate (glucose or fructose), $5.0 \%$ corn oil, $5.0 \%$ cellulose, $3.5 \%$ AIN93G mineral mixture, $1.0 \%$ AIN93 vitamin mixture, $0.3 \%$ l-cystine, and $0.2 \%$ choline chloride. The animals were allowed free access to diets and water for 12 days. At the end of the feeding period, the rats were fasted for $7 \mathrm{~h}$ and euthanized by cardiac puncture under isoflurane anesthesia. Blood and liver tissues were collected for subsequent assays. All animal care and experimental procedures were approved by the Gifu University Animal Care and Usage Committee.

Blood was centrifuged at $1,710 \times g$ for $15 \mathrm{~min}$ at $4{ }^{\circ} \mathrm{C}$ to prepare serum. Serum concentrations of triglycerides, total cholesterol, and high-density lipoprotein (HDL)-cholesterol were measured using commercial kits (Triglyceride E-test Wako, Cholesterol E-test Wako, and HDL-Cholesterol E-test Wako, respectively; FUJIFILM Wako Pure Chemical Corporation, Osaka, Japan). Serum VLDL+low-density lipoprotein (LDL)-cholesterol was determined as follows: total cholesterol - HDL-cholesterol.

Total lipids from liver tissues were extracted by the Bligh and Dyer method (Bligh and Dyer 1959) with some modifications. Liver tissues were homogenized with $0.1 \mathrm{M} \mathrm{KCl}$ and mixed with chloroform and methanol at a ratio of $1: 1: 1$. The homogenates were centrifuged at $800 \times g$ for $10 \mathrm{~min}$ at room temperature. Then, the lower organic phase was collected and dried as lipid extracts. The levels of triglycerides and cholesterol in the lipid extracts were dissolved in 2-propanol and measured using a commercial kit (Triglyceride E-test Wako and Cholesterol E-test Wako, respectively; FUJIFILM Wako Pure Chemical Corporation).

Hepatic total RNA was extracted and converted to cDNA using commercial kits (RNeasy Mini kit; Qiagen, Tokyo, Japan and ReverTra Ace qPCR RT kit; Toyobo, Osaka, Japan, respectively). PCR amplification was performed in a final volume of $20 \mu \mathrm{L}$, which contained SYBR Green (TB Green Premix Ex Taq; Takara, Shiga, Japan), $0.2 \mu \mathrm{M}$ of each primer, Rox reference dye, and $20 \mathrm{ng}$ of cDNA, using a real-time PCR system (StepOnePlus; Applied Biosystems Japan, Tokyo, Japan). The primers used were listed in Table 1. Relative mRNA levels were determined by the $\Delta \Delta \mathrm{Ct}$ method (Livak and Schmittgen 2001), using ribosomal protein, large, $\mathrm{P} 0(\operatorname{Rplp} 0)$ as a normalization control.

Liver tissues were homogenized in radioimmunoprecipitation buffer $(50 \mathrm{mM}$ tris- $\mathrm{HCl} \mathrm{pH} 8.0$, $150 \mathrm{mM} \mathrm{NaCl}, 0.5 \%$ sodium deoxycholate, $0.1 \%$ SDS, $1.0 \%$ Nonidet P-40) containing protease inhibitors (Complete Mini; Sigma-Aldrich Japan, Tokyo, Japan). The homogenates were centrifuged at $16,100 \times g$ for $30 \mathrm{~min}$ at $4{ }^{\circ} \mathrm{C}$ to prepare lysates. Protein concentrations

Table 1. Sequences of primers

Forward

Reverse

$\begin{array}{ll}\text { Hmgcr } & \text { 5'-TCTGCAGTACCTGCCTTACAGA-3' } \\ \text { Ldlr } & \text { 5'-CCCAACCTGAAGAATGTGGT-3' } \\ \text { Acat1 } & \text { 5'-GTCCATGGAGGAGCTGTTTC-3' } \\ \text { Acat2 } & \text { 5'-CCAAAGTGGCTCCAGAAGAG-3' } \\ \text { Mttp } & \text { 5'-TCTTCCAGTACATCGGAAAGG-3' } \\ \text { Apob } & \text { 5'-GATGGAGATGGGAGATGAGGT-3' } \\ \text { Cyp7al } & \text { 5'-CAAGACGCACCTCGCTATTC-3' } \\ \text { Abcg5 } & \text { 5'-GAATGTGTCCTTCAGCGTCA-3' } \\ \text { Abcg8 } & \text { 5'-AACCCTGCTGACTTCTACGTG-3' } \\ \text { Aldob } & \text { 5'-TTGCCAATGGGAAGGGTA-3' } \\ \text { Rplp0 } & \text { 5'-CGAGAAGACCTCTTTCTTCCAA-3' }\end{array}$
5'-ATCACGTTCTCACAGCAAGC-3'
5'-TGGCATCATAGGACAAGCTG-3'
5'-AAGGCATGAGCCAAGTGAAC-3'
5'-CCACACTGGCTTGTCGAGTA-3'
5'-GTGGCTGCAATTAAGCCTTC-3'
5'- GGGCTCCTCATCAACAAGAG-3'
5'-CTGCTTTCATTGCTTCAGGA-3'
5'-GCTGGCATGATTTGATGTTC-3'
5'-TGCAAGTAATCGAGCCTTCTC-3'
5'-ATCCTCTGTAGGCGGTTTCC-3'
5'-AGTCTTTATCAGCTGCACATCG-3' 
in the lysates were determined by a bicinchoninic acid assay (BCA protein assay kit; Takara). The lysates containing an equal amount of protein $(40 \mu \mathrm{g})$ were separated by $12.5 \%$ SDS-PAGE, and then transferred onto PVDF membranes. After transfer, the membranes were washed in phosphate-buffered saline (PBS) with $0.1 \%$ tween 20 (PBS-T) and blocked in 3-5\% skimmed milk in PBS-T for $1 \mathrm{~h}$ at room temperature. Thereafter, the membranes were incubated overnight at $4{ }^{\circ} \mathrm{C}$ with the following primary antibodies: anti-ACAT2 (Santa Cruz Biotechnology, Dallas, TX, USA), anti-aldolase, fructose-bisphosphate B (ALDOB) (GeneTex, Irvine, CA, USA), and anti-TATA-binding protein (TBP) (GeneTex). Subsequently, the membranes were washed in PBS-T and incubated for $1 \mathrm{~h}$ at $4{ }^{\circ} \mathrm{C}$ with the secondary antibody conjugated with horseradish peroxidase (Cell Signaling Technology Japan, Tokyo, Japan). After washing with PBS-T, the signals were detected by enhanced chemiluminescence (Immnostar LD; FUJIFILM Wako Pure Chemical Corporation) using an imaging system (LAS3000; Fujifilm, Tokyo, Japan). Relative protein levels were normalized using TBP as a loading control.

Values are expressed as means \pm SEM. Differences between the two groups were evaluated by the Student's $t$-test. $P<0.05$ indicated statistical significance.

Body weight, food intake, and serum HDL-cholesterol levels did not differ between groups.
Hepatic triglyceride and cholesterol levels and serum levels of triglycerides, total cholesterol, and VLDL+LDL-cholesterol were significantly higher in the fructose group than in the glucose group (Table 2).

Hepatic expression levels of $\mathrm{Hmgcr}$, LDL receptor $(L d l r)$, acetyl-coenzyme A acetyltransferase 1 (Acat1), Mttp, Apob, and cytochrome P450 family 7 subfamily A member 1 (Cyp7al) were not different between groups. These genes encode proteins involved in LDL update from the blood (LDLR) and cholesterol esterification (ACAT), and the rate limiting enzyme for cholesterol catabolism and bile acid synthesis (CYP7A1). Hepatic levels of ATP binding cassette subfamily G member 5 (Abcg5) were significantly lower $(P<0.05)$ in the fructose group and $A b c g 8$ was also lower $(P=0.057)$. ABCG5 and ABCG8 form a heterodimer and are involved in the excretion of cholesterol in bile. In contrast, hepatic expression of ACAT2 and ALDOB was significantly higher at the gene and protein levels in the fructose group (Fig. 1A, 1B). ALDOB is a key enzyme involved in fructolysis and is induced by high levels of fructose (Koo et al. 2008, Shimada et al. 2017).

High levels of fructose induce hypertriglyceridemia. In this study, we observed higher levels of serum triglycerides and VLDL+LDL-cholesterol in rats fed a high-fructose diet compared to those in animals fed a high-glucose diet. These results were consistent with those of previous studies using rats (Koo et al. 2008, Shimada et al. 2017). Interestingly, in rats fed a high-

Table 2. Physiological and biochemical parameters of rats fed a glucose diet or a fructose diet for 12 days

\begin{tabular}{|c|c|c|c|c|}
\hline & Glucose & \multicolumn{3}{|c|}{ Fructose } \\
\hline Body weight & & & & \\
\hline Initial $(g)$ & $122 \pm 3$ & 120 & \pm & 3 \\
\hline Final $(g)$ & $171 \pm 4$ & 164 & \pm & 3 \\
\hline Food intake $(g / d)$ & $12.9 \pm 0.4$ & 12.0 & \pm & 0.3 \\
\hline \multicolumn{5}{|l|}{ Liver } \\
\hline Weight $(g)$ & $7.23 \pm 0.26$ & 8.60 & \pm & $0.35^{* *}$ \\
\hline Triglycerides ( $\mathrm{mg} / \mathrm{g}$ liver) & $51 \pm 23$ & 135 & \pm & $19^{*}$ \\
\hline Cholesterol (mg/g liver) & $7.06 \pm 1.05$ & 9.81 & \pm & $0.59^{*}$ \\
\hline \multicolumn{5}{|l|}{ Serum } \\
\hline Triglycerides ( $\mathrm{mg} / 100 \mathrm{ml})$ & $130 \pm 8$ & 254 & \pm & $29^{* *}$ \\
\hline Total cholesterol $(\mathrm{mg} / 100 \mathrm{ml})$ & $75 \pm 3$ & 103 & \pm & $4^{* *}$ \\
\hline$V L D L+L D L$-cholesterol $(\mathrm{mg} / 100 \mathrm{ml})$ & $37.6 \pm 2.4$ & 58.5 & \pm & $4.9^{* *}$ \\
\hline HDL-cholesterol $(\mathrm{mg} / 100 \mathrm{ml})$ & $37.8 \pm 3.5$ & 44.9 & \pm & 2.9 \\
\hline
\end{tabular}

Values are expressed as means $\pm \mathrm{SEM}(\mathrm{n}=7) . * * p<0.01, * P<0.05$ 
(A)

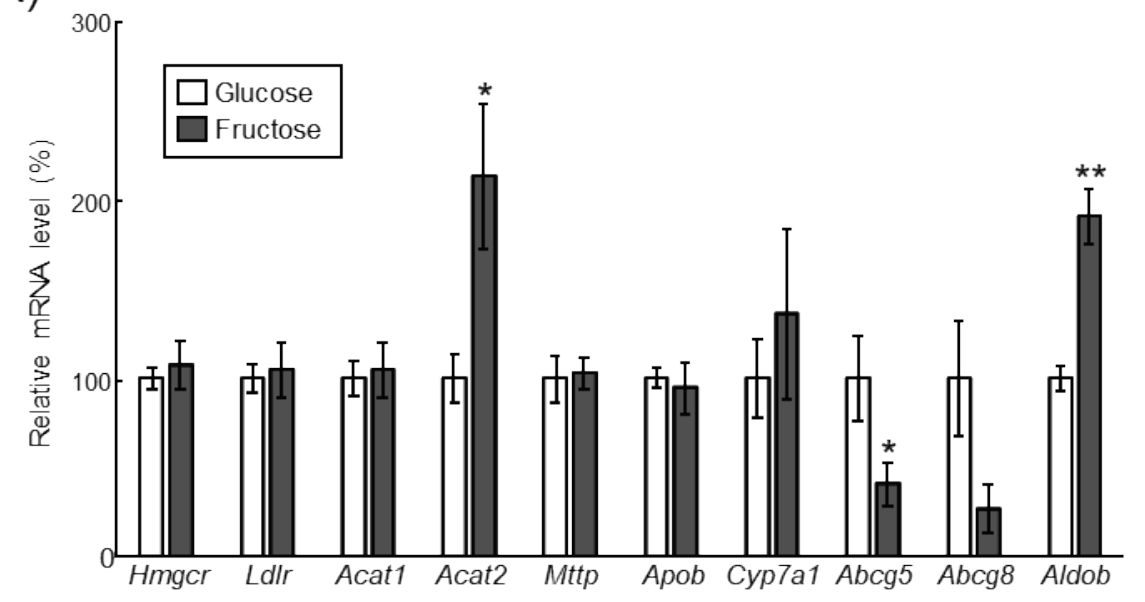

(B)
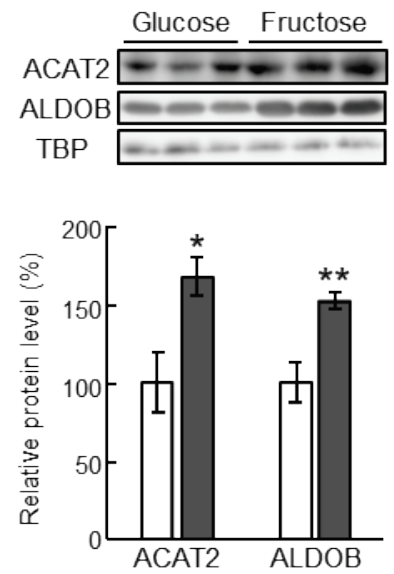

Fig. 1. Hepatic expression of cholesterol metabolism-related genes and proteins in rats fed a glucose or fructose diet. (A) Expression of cholesterol metabolism-related genes. (B) Expression of ACAT2 and ALDOB proteins. Values are expressed as means \pm SEM ( $n=7$ ). $* * P<0.01, * P<0.05$.

fructose diet, we observed an increase in the hepatic expression of ACAT2 at both gene and protein levels. This is the first study to show that feeding rats a highfructose diet induces hepatic expression of ACAT2 and hypertriglyceridemia. ACAT2 is predominantly expressed in livers and intestines, whereas ACAT1 is ubiquitously expressed (Anderson et al. 1998, Cases et al. 1998, Oelkers et al. 1998). Especially in livers, ACAT2 is localized in hepatocytes, which play a main role in nutritional metabolism, whereas ACAT1 is localized in Kupffer cells, which play a key role in the removal of foreign bodies (Parini et al. 2010). In addition, Liang et al. (2004) reported that the assembly/secretion of VLDL is more strongly induced in rat hepatocytes overexpressing human Acat2 than those expressing Acat1. Moreover, several studies showed that Acat2 deficiency or inhibition prevents atherosclerosis (Willner et al. 2003, Ohshiro et al. 2011), whereas Acat1 deficiency had no effect, in atherosclerotic mice model (Accad et al. 2000, Fazio et al. 2001). Considering our results and aforementioned reports, the high fructoseinduced hepatic expression of ACAT2, but not ACAT1, might lead hypertriglyceridemia and promote subsequent vascular diseases such as atherosclerosis via lipid metabolic abnormalities in the liver. However, it is unclear how fructose enhances the expression of Acat 2 in the liver. The present data indicate that feeding rats a high-fructose diet also induces hepatic expression of ALDOB and ACAT2 at the gene and protein levels. In addition, several studies have demonstrated that the transcription factor hepatocyte nuclear factor 1 (HNF1) regulates both Aldob and Acat2 (Gregori et al. 2002, Pramfalk et al. 2005). Therefore, high levels of fructose might increase Acat2 expression via the activation of HNF1 in rat livers. Further studies should investigate whether high fructose levels induce the recruitment of HNF1 to the Acat2 promoter.

It should be noted that high-fructose feeding resulted in higher cholesterol and lower Abcg5/8 expression in the liver. Su et al. (2012) reported that higher levels of plasma cholesterol and triglycerides are observed in $A b c g 5 / 8$-knockout mice fed a high-fat diet. Considering the role of $\mathrm{ABCG} 5 / 8$ in the excretion of cholesterol from the liver to bile, it is likely that a fructose-induced increase in hepatic cholesterol is partially responsible for the lower $A b c g 5 / 8$ expression, which might lead to subsequent hyperlipidemia. This hypothesis requires further investigation.

In conclusion, our results suggest that hypertriglyceridemia induced by a high-fructose diet is associated with increased ACAT2 expression at both gene and protein levels in the liver. This finding has implications for the prevention and treatment of metabolic diseases such as fatty liver and obesity.

\section{Conflict of Interest}

There is no conflict of interest.

\section{Acknowledgements}

This work was supported by JSPS KAKENHI Grant number JP18K02237. 


\section{References}

ACCAD M, SMITH SJ, NEWLAND DL, SANAN DA, KING LE JR, LINTON MF, FAZIO S, FARESE RV JR: Massive xanthomatosis and altered composition of atherosclerotic lesions in hyperlipidemic mice lacking acyl CoA:cholesterol acyltransferase 1. J Clin Invest 105: 711-719, 2000.

ANDERSON RA, JOYCE C, DAVIS M, REAGAN JW, CLARK M, SHELNESS GS, RUDEL LL: Identification of a form of acyl-CoA:cholesterol acyltransferase specific to liver and intestine in nonhuman primates. $J$ Biol Chem 273: 26747-26754, 1998.

BLIGH EG, DYER WJ: A rapid method of total lipid extraction and purification. Can J Biochem Physiol 37: 911-917, 1959.

CASES S, NOVAK S, ZHENG YW, MYERS HM, LEAR SR, SANDE E, WELCH CB, LUSIS AJ, SPENCER TA, KRAUSE BR, ERICKSON SK, FARESE RV JR: ACAT-2, a second mammalian acyl-CoA: cholesterol acyltransferase. Its cloning, expression, and characterization. J Biol Chem 273: 26755-26764, 1998.

FAZIO S, MAJOR AS, SWIFT LL, GLEAVES LA, ACCAD M, LINTON MF, FARESE RV JR: Increased atherosclerosis in LDL receptor-null mice lacking ACAT1 in macrophages. J Clin Invest 107: 163-171, 2001.

GREGORI C, PORTEU A, MITCHELL C, KAHN A, PICHARD AL: In vivo functional characterization of the aldolase B gene enhancer. J Biol Chem 277: 28618-28623, 2002.

HERMAN MA, SAMUEL VT: The Sweet path to metabolic demise: fructose and lipid synthesis. Trends Endocrinol Metab 27: 719-730, 2016.

JANEVSKI M, RATNAYAKE S, SILJANOVSKI S, MCGLYNN MA, CAMERON-SMITH D, LEWANDOWSKI P: Fructose containing sugars modulate mRNA of lipogenic genes ACC and FAS and protein levels of transcription factors ChREBP and SREBP1c with no effect on body weight or liver fat. Food Funct 3: 141-149, 2012.

KOO HY, WALLIG MA, CHUNG BH, NARA TY, CHO BH, NAKAMURA MT: Dietary fructose induces a wide range of genes with distinct shift in carbohydrate and lipid metabolism in fed and fasted rat liver. Biochim Biophys Acta 1782: 341-348, 2008.

LIANG JJ, OELKERS P, GUO C, CHU PC, DIXON JL, GINSBERG HN, STURLEY SL: Overexpression of human diacylglycerol acyltransferase 1, acyl-coa:cholesterol acyltransferase 1, or acyl-CoA:cholesterol acyltransferase 2 stimulates secretion of apolipoprotein B-containing lipoproteins in McA-RH7777 cells. J Biol Chem 279: 44938-44944, 2004.

LIVAK KJ, SCHMITTGEN TD. Analysis of relative gene expression data using real-time quantitative PCR and the 2(-Delta Delta C(T)) method. Methods 25: 402-408, 2001.

OELKERS P, BEHARI A, CROMLEY D, BILLHEIMER JT, STURLEY SL: Characterization of two human genes encoding acyl coenzyme A: cholesterol acyltransferase-related enzymes. J Biol Chem 273: 26765-26771, 1998.

OHSHIRO T, MATSUDA D, SAKAI K, DEGIROLAMO C, YAGYU H, RUDEL LL, OMURA S, ISHIBASHI S, TOMODA H: Pyripyropene A, an acyl-coenzyme A: cholesterol acyltransferase 2-selective inhibitor, attenuates hypercholesterolemia and atherosclerosis in murine models of hyperlipidemia. Arterioscler Thromb Vasc Biol 31: 1108-1115, 2011.

PARINI P, DAVIS M, LADA AT, ERICKSON SK, WRIGHT TL, GUSTAFSSON U, SAHLIN S, EINARSSON C, ERIKSSON M, ANGELIN B, TOMODA H, OMURA S, WILLINGHAM MC, RUDEL LL. ACAT2 is localized to hepatocytes and is the major cholesterol-esterifying enzyme in human liver. Circulation 110: 2017-2023, 2004.

PRAMFALK C, DAVIS MA, ERIKSSON M, RUDEL LL, PARINI P: Control of ACAT2 liver expression by HNF1. J Lipid Res 46: 1868-1876, 2005.

SHIMADA M, HIBINO M, TAKESHITA A: Dietary supplementation with myo-inositol reduces hepatic triglyceride accumulation and expression of both fructolytic and lipogenic genes in rats fed a high-fructose diet. Nutr Res 47: 21-27, 2017. 
SHIMADA M, ICHIGO Y, SHIROUCHI B, TAKASHIMA S, INAGAKI M, NAKAGAWA T, HAYAKAWA T: Treatment with myo-inositol attenuates binding of the carbohydrate-responsive element-binding protein to the ChREBP- $\beta$ and FASN genes in rat nonalcoholic fatty liver induced by high-fructose diet. Nutr Res 64: 49-55, 2019.

SU K, SABEVA NS, LIU J, WANG Y, BHATNAGAR S, VAN DER WESTHUYZEN DR, GRAF GA. The ABCG5 ABCG8 sterol transporter opposes the development of fatty liver disease and loss of glycemic control independently of phytosterol accumulation. J Biol Chem 287: 28564-28575, 2012.

WILLNER EL, TOW B, BUHMAN KK, WILSON M, SANAN DA, RUDEL LL, FARESE RV JR: Deficiency of acyl CoA:cholesterol acyltransferase 2 prevents atherosclerosis in apolipoprotein E-deficient mice. Proc Natl Acad Sci US A 100: 1262-1267, 2003. 